\section{Óbitos infantis evitáveis em hospital de referência estadual do Nordeste brasileiro}

\author{
Avoidable infant deaths at a reference \\ hospital in Northeast Brazil
}

Suely Arruda Vidal 1

Paulo Germano de Frias 2

Flora Morais Pais Barreto 3

Lygia Carmen Morais Vanderlei 4

Eronildo Felisberto 5

1-5 Instituto Materno Infantil de Pernambuco, IMIP. Rua dos Coelhos, 300. Recife, PE, Brasil. 50.070-550. crianca@imip.org.br

\begin{abstract}
Objectives: to classify deaths of children under one year old from January to December 2000, at a public Hospital of Pernambuco, according to avoidable basic causes of death.

Methods: descriptive cross sectional study using data from the Information System on Mortality processed at the Hospital. The basic causes stated in Death Certificates collected from the medical records and codified under the rules of the Tenth International Disease Classification were grouped according to the criteria proposed by the SEADE Foundation (1991). The coefficient of hospital infant mortality was calculated based on the hospital admission files.

Results: the majority of deaths were classified as deaths avoidable by adequate healthcare, with proportional mortality rates of $77,4 \%$ for infant deaths, $75,3 \%$ for neonatal deaths and 60,1\% for post-neonatal deaths, representing an infant death rate of 103,7 deaths per 1.000 hospital admissions. Approximately half were neonates, of these $31 \%$ were avoidable through early diagnosis and care. In the post-neonatal group, $44 \%$ were principally caused by intestinal infectious diseases and respiratory diseases.

Conclusions: the results are suggestive that these high percentages of avoidable deaths were caused by difficulties of access to healthcare services, coverage and/or quality of the assistance.
\end{abstract}

Key words Infant mortality, Hospital mortality, Cause of death

\section{Resumo}

Objetivos: classificar os óbitos de menores de um ano ocorridos de janeiro a dezembro de 2000, em hospital de referência em Pernambuco, segundo critérios de evitabilidade da causa básica.

Métodos: estudo descritivo de corte transversal, utilizando-se como fonte de dados o Sistema de Informação sobre Mortalidade, processado na instituição. As causas básicas presentes nas Declarações de Óbitos, corrigidas a partir do prontuário médico e codificadas segundo as regras da 10 revisão da Classificação Internacional de Doenças, foram agrupadas segundo os critérios de evitabilidade de óbitos propostos pela Fundação SEADE em 1991. Calculou-se o coeficiente de mortalidade infantil hospitalar.

Resultados: a maioria dos óbitos estava classificada entre as causas reduzíveis por medidas de atenção à saúde, com mortalidade proporcional de $77,4 \%$ (infantil), 75,3\% (neonatal) e 60,1\% (pósneonatal) representando um coeficiente de mortalidade infantil hospitalar de 103,7 óbitos por 1.000 internações. Quase metade era neonatos, desses, $31 \%$ eram reduzíveis por diagnóstico e tratamento precoces. No grupo pós-neonatal, 44\% foram causadas principalmente pelas doenças infecciosas intestinais e do aparelho respiratório.

Conclusões: os altos percentuais de mortes por causas evitáveis, sugerem problemas de acesso aos serviços de saúde, cobertura elou na qualidade da assistência prestada.

Palavras-chave Mortalidade infantil, Mortalidade hospitalar, Causa da morte 


\section{Introdução}

A mortalidade infantil é um indicador clássico de saúde de uma população, sendo também considerada um evento traçador da qualidade dos serviços. ${ }^{1-4}$ As precárias condições socioeconômicas e ambientais, aliadas à dificuldade de acesso aos serviços de saúde constituem os principais fatores condicionantes e/ou determinantes dos óbitos infantis.

Esse indicador apresenta valores variáveis, inversamente proporcionais ao grau de desenvolvimento da região. Porém, independente dos valores encontrados, vários trabalhos apontam para um declínio do coeficiente de mortalidade infantil (CMI) no Brasil, nas três últimas décadas. ${ }^{5-11}$

Essa queda tem sido mais evidente no componente pós-neonatal, por ser esse mais susceptível às ações de promoção, prevenção, diagnóstico e tratamento precoce, mediante o emprego de tecnologias simplificadas e de baixo custo, além da melhoria das condições ambientais. 4,12

Já o componente neonatal tem mostrado um decréscimo mais lento, provavelmente por estar associado tanto a fatores biológicos, de prevenção mais difícil, como ao acesso e à qualidade da assistência prénatal, ao parto e ao recém-nascido, uma vez que reflete melhor a qualidade da atenção à saúde recebida pelas crianças e mães do que o bem-estar social. ${ }^{4}$ As intervenções nessa faixa etária, por serem mais complexas, necessitam de investimentos em serviços hospitalares; entretanto procedimentos rotineiros e simples de atenção ao pré-natal e ao parto parecem não estar sendo aplicados adequadamente, sugerindo uma incapacidade dos serviços de incorporarem tecnologias simples e eficazes. 3,13

Pernambuco, na região Nordeste, segue a tendência do país, apresentando diminuição da mortalidade infantil, porém com coeficientes maiores que os estados do Sul e Sudeste. Nessas regiões o CMI em 1990 foi 28,7 e 30,2\%o nascidos vivos (nv) reduzindo-se para 19,7 e 20,6\% nv respectivamente, em 2000,14 enquanto o Nordeste apresentou, nesses mesmos anos, CMI de 73 e $44 \%$ nv; dentro dessa região, o CMI para Pernambuco foi de 58,2\%o nv em 1999.14,15 Tais números explicitam a persistência de importantes diferenciais de mortalidade no país, compatíveis com as desigualdades socioeconômicas inter-regionais. No entanto, esses coeficientes poderiam ser reduzidos, considerando-se o perfil de mortalidade e a possibilidade de se evitar o óbito nas diversas etapas do processo de adoecimento dessas crianças.

O conceito de evitabilidade das causas, seja por razões socioeconômicas ou por políticas públicas do setor saúde, vem sendo bastante discutido e as proposições de classificação apresentadas têm, cada uma, vantagens e limitações, variando de acordo com a questão e o foco.1,16-18 O grau de subjetividade, a fonte dos dados, o processo de trabalho, entre outros aspectos metodológicos, variam em cada classificação.

Alguns estudos sobre o assunto têm utilizado os critérios de classificação da Fundação Sistema Estadual de Análise de Dados (FSEADE) 3,17,19-22 que se baseiam no conhecimento técnico-científico existente, para evitar o aparecimento de determinadas doenças, seu agravamento e o óbito, independente do fato de as tecnologias estarem ou não acessíveis para a maioria da população naquele momento. ${ }^{12,17}$

A abordagem do tema em hospitais de maior nível de complexidade, onde parte considerável dos casos graves expressa a inadequação da assistência em outros níveis do sistema de saúde, pode trazer à luz reflexões que contribuam para a reversão do quadro.

O estudo da mortalidade infantil hospitalar se justifica por ser uma ferramenta útil para evidenciar problemas na qualidade da assistência, nas condições em que a morte não é um evento raro, 23 situação em que se insere o presente trabalho. O seu objetivo é classificar os óbitos dos menores de um ano do Instituto Materno Infantil de Pernambuco, ocorridos no período de janeiro a dezembro de 2000 , - segundo os critérios de evitabilidade da Fundação SEADE, - a fim de fornecer subsídios aos gestores institucionais hospitalares como também para as instâncias governamentais.

\section{Métodos}

Foi realizado um estudo descritivo de corte transversal no Hospital Geral de Pediatria do Instituto Materno Infantil de Pernambuco (IMIP), em Recife, PE, Brasil, considerando o universo dos óbitos não fetais de menores de um ano ocorridos no período de janeiro a dezembro de 2000.

O IMIP é um complexo hospitalar de nível terciário, composto pelo Hospital Geral de Pediatria (HGP) e pelo Centro de Atenção à Mulher (CAM). Está localizado no I Distrito Sanitário do Recife, fazendo parte da rede complementar do SUS, onde realizam em torno de 500 partos, aproximadamente 20.000 atendimentos pediátricos ambulatoriais, e em média, 1.200 internações mensais de crianças em todas as suas faixas etárias, dispondo de leitos para internação clínica, isolamento infantil, cirurgia pediátrica e UTI pediátrica. O HGP é de referência estadual e regional para as doenças de maior com- 
plexidade, incluindo as malformações congênitas passíveis de correção cirúrgica.

No setor de internação clínica do HGP são admitidas crianças transferidas de outros serviços ou procedentes do domicílio, existindo enfermarias exclusivas para crianças no período neonatal, pósneonatal e para as demais faixas etárias, com limite até os dezesseis anos.

A fonte de dados utilizada foi o banco do Sistema de Informação sobre Mortalidade (SIM) do Ministério da Saúde processado na instituição. As causas básicas, contidas nas Declarações de Óbitos (DO), corrigidas sistematicamente por uma equipe de epidemiologistas, pediatras e neonatologistas, a partir do prontuário médico, foram codificadas segundo as regras da $10^{a}$ revisão da Classificação Internacional de Doenças24 (CID 10) e a seguir, agrupadas pelos critérios de evitabilidade das doenças propostos pela Fundação SEADE, 17 adaptados da $9^{\text {a }}$ revisão da CID para a CID 10.

A mortalidade infantil hospitalar proporcional por componentes, neonatal e pós-neonatal, bem como os respectivos coeficientes de mortalidade foram analisados segundo classificação de evitabilidade das causas, definida pela existência de medidas de atenção à saúde.

Para o cálculo dos coeficientes de mortalidade infantil e de seus componentes, neonatal e pósneonatal, utilizou-se a razão entre o número de mortes de menores de um ano que ocorreram no HGP por grupo etário e o total de internamentos de menores de um ano, representando os expostos ao risco multiplicado por 1.000 . O número de interna- mentos foi obtido através do Sistema de Informação Hospitalar do Sistema Único de Saúde (SIH/SUS), no setor de informática do IMIP, totalizando 3.656 internações de menores de um ano em 2000.

Foram calculados os coeficientes de mortalidade hospitalar por causas "reduzíveis" e "outras situações", segundo a classificação da FSEADE, 17 a saber: 1) reduzíveis por imunoprevenção, por adequado controle da gravidez, por adequada atenção ao parto, por diagnóstico e tratamento precoces, parcialmente reduzíveis e outras causas reduzíveis; 2) outras situações: não evitáveis, desconhecidas e outras causas não classificadas. Do total de 588 óbitos de crianças menores de um ano ocorridos no Hospital Geral de Pediatria do IMIP, em 2000, foram investigados 564, após exclusão dos enviados ao Serviço de Verificação de Óbitos (SVO), em número de 24.

\section{Resultados}

Observou-se que praticamente a metade $(46,6 \%)$ encontrava-se na faixa etária neonatal.

Analisando-se os coeficientes de mortalidade infantil e a mortalidade proporcional segundo causa básica verificou-se que a principal causa correspondeu as constantes do capítulo "Afecções perinatais" da CID10 (33,2\%), com um Coeficiente de Mortalidade Infantil Hospitalar (CMIH) de 51 por 1.000 internações. A segunda pertenceu ao grupo das "Malformações congênitas" (24,3\%) e CMIH de 37,4 por 1.000 internações. As doenças infecciosas intestinais apareceram em terceiro lugar $(16,8 \%)$, com CMIH de 26 por 1.000 internações (Tabela 1).

Tabela 1

Mortalidade proporcional e coeficientes de mortalidade infantil hospitalar por mil internações segundo causa básica de óbito no Hospital Geral de Pediatria do Instituto Materno Infantil de Pernambuco, 2000.

\begin{tabular}{lccc}
\hline & Mortalidade proporcional & Coeficiente de Mortalidade Infantil Hopitalar \\
\cline { 2 - 4 } Causas & $n$ & $\%$ & $\% 0$ \\
\hline Afeç̧ões perinatais & 187 & 33,2 & 51,1 \\
Malformações congênitas & 137 & 24,3 & 37,4 \\
Doenças infecciosas intestinais & 95 & 16,8 & 26,0 \\
Pneumonias & 47 & 8,3 & 12,8 \\
Desnutrição & 17 & 3,0 & 4,6 \\
Meningite & 10 & 1,8 & 2,7 \\
Demais causas & 70 & 12,4 & 19,1 \\
Sintomas, sinais ...** & 1 & 0,2 & 0,3 \\
Total & 564 & 100,0 & 154,3 \\
\hline
\end{tabular}

* Coeficiente de mortalidade infantil hospitalar = razão entre o $n .^{\circ}$ de óbitos em menores de um ano e o total de internamentos (3.656) na mesma faixa etária multiplicado por 1000; ** Sinais, sintomas e achados anormais nos exames.24 
Classificando-se as causas básicas segundo os critérios de evitabilidade por medidas de atenção à saúde identificou-se que, tanto os óbitos neonatais precoces (de 0 a 6 dias) quanto os tardios (7 a 27 dias), apresentaram maior proporção para as reduzíveis por diagnóstico e tratamento precoces, $28,0 \%$ e $38,0 \%$ respectivamente, com coeficiente de mortalidade neonatal hospitalar de 22,7 por 1.000 internações. Agrupando-se todas as reduzíveis encontrou-se, um total de 263 óbitos ocorridos no primeiro mês de vida, dos quais 198 (75\%) poderiam ter sido evitados (Tabela 2).

Quanto aos óbitos pós-neonatais, aproximadamente dois terços $(60,1 \%)$, aconteceram por causas reduzíveis, estando a maioria classificada entre as "Outras reduzíveis", com mortalidade proporcional e coeficiente de mortalidade pós-neonatal hospitalar de $44,2 \%$ e 36,41 por 1.000 internações. Encontrouse um percentual de $10,0 \%$ das causas reduzíveis por diagnóstico e tratamento precoces, representando um coeficiente de 8,2 por 1.000 internações. Das 301 mortes ocorridas nesse período de vida, 181 poderiam ter sido evitadas por medidas de atenção à saúde (Tabela 3).

A Tabela 4 apresenta os coeficientes de mortalidade infantil hospitalar e a mortalidade proporcional segundo a classificação de evitabilidade da causa básica. A maioria dos óbitos encontra-se entre as causas reduzíveis por medidas de atenção à saúde, com mortalidade proporcional de $77,4 \%$ para os infantis, $75,3 \%$, neonatais e $60,1 \%$, pós-neonatais. Os coeficientes de mortalidade por essas causas atingiram 103,7; 54,2 e 49,5 óbitos por 1.000 internações para os infantis, neonatais e pós-neonatais, respectivamente. Esses coeficientes traduziram em número absoluto, 379 óbitos do total de 564. As causas de mortes dos menores de um ano enquadradas em "Outras situações", correspondentes as não evitáveis, desconhecidas e outras causas, apresentaram coeficiente de 50,6 óbitos por 1.000 internações.

Tabela 2

Mortalidade proporcional por grupo etário e coeficiente de mortalidade neonatal hospitalar, segundo classificação de redutibilidade da causa básica por medidas de atenção à saúde. Hospital Geral de Pediatria do Instituto Materno Infantil de Pernambuco, 2000.

\begin{tabular}{|c|c|c|c|c|c|c|c|}
\hline \multirow[b]{2}{*}{ Classificação } & \multicolumn{2}{|c|}{$0-6$ dias } & \multicolumn{2}{|c|}{$7-27$ dias } & \multicolumn{2}{|c|}{ Total } & \multirow{2}{*}{$\begin{array}{c}\text { Coeficiente de } \\
\text { Mortalidade Infantil } \\
\text { Hospitalar* }\end{array}$} \\
\hline & $\mathrm{n}$ & $\%$ & $\mathrm{n}$ & $\%$ & $\mathrm{n}$ & $\%$ & \\
\hline \multicolumn{8}{|l|}{ Reduzíveis } \\
\hline Por adequado controle da gravidez & 9 & 5,4 & 12 & 12,6 & 21 & 8,0 & 5,7 \\
\hline Por adequada atenção ao parto & 14 & 8,4 & 2 & 2,1 & 16 & 6,1 & 4,4 \\
\hline Por diagnóstico e tratamento precoces & 47 & 28,0 & 36 & 38,0 & 83 & 31,5 & 22,7 \\
\hline Parcialmente reduzíveis & 44 & 26,2 & 2 & 2,1 & 46 & 17,5 & 12,6 \\
\hline Outras reduzíveis & 18 & 10,7 & 14 & 14,7 & 32 & 12,2 & 8,7 \\
\hline Sub-total & 132 & 78,6 & 66 & 69,5 & 198 & 75,3 & 54,2 \\
\hline \multicolumn{8}{|l|}{ Outras situações } \\
\hline Não evitáveis & 27 & 16,1 & 27 & 28,4 & 54 & 20,5 & 14,8 \\
\hline Desconhecidas & 0 & - & 0 & - & 0 & - & - \\
\hline Outras causas & 9 & 5,3 & 2 & 2,1 & 11 & 4,2 & 3,0 \\
\hline Sub-total & 36 & 21,4 & 29 & 30,5 & 65 & 24,7 & 17,8 \\
\hline Total & 168 & 100,0 & 95 & 100,0 & 263 & 100,0 & 71,9 \\
\hline
\end{tabular}

* Coeficiente de mortalidade infantil hospitalar = razão entre o n. ${ }^{\circ}$ de óbitos em menores de 28 dias e o total de internamentos (3.656) multiplicado por 1000 
Mortalidade proporcional e coeficiente de mortalidade pós-neonatal hospitalar segundo classificação de redutibilidade da causa básica por medidas de atenção à saúde. Hospital Geral de Pediatria do Instituto Materno Infantil de Pernambuco, 2000.

\begin{tabular}{|c|c|c|c|}
\hline \multirow{2}{*}{ Classificação } & \multicolumn{2}{|c|}{ Mortalidade proporcional } & \multirow{2}{*}{$\begin{array}{c}\text { Coeficiente de Mortalidade Infantil Hopitalar* } \\
\% \%\end{array}$} \\
\hline & $\mathrm{n}$ & $\%$ & \\
\hline \multicolumn{4}{|l|}{ Reduzível } \\
\hline Por prevenção & 1 & 0,3 & - \\
\hline Por diagnóstico e tratamento precoces & 30 & 10,0 & 8,2 \\
\hline Parcialmente reduzíveis & 17 & 5,6 & 4,6 \\
\hline Outras reduzíveis & 133 & 44,2 & 36,4 \\
\hline Sub-total & 181 & 60,1 & 49,5 \\
\hline \multicolumn{4}{|l|}{ Outras situações } \\
\hline Não evitáveis & 88 & 29,2 & 24,1 \\
\hline Desconhecidas & 1 & 0,3 & - \\
\hline Outras causas & 31 & 10,4 & 8,5 \\
\hline Sub-total & 120 & 39,9 & 32,8 \\
\hline Total & 301 & 100,0 & 82,3 \\
\hline
\end{tabular}

* Coeficiente de mortalidade infantil hospitalar = razão entre o $n .^{\circ}$ de óbitos em menores de um ano e maiores de 27 dias e o total de internamentos (3.656) multiplicado por 1.000

Tabela 4

Mortalidade proporcional e coeficientes de mortalidade neonatal, pós-neonatal e infantil hospitalar segundo classificação de redutibilidade da causa básica por medidas de atenção à saúde. Hospital Geral de Pediatria do Instituto Materno Infantil de Pernambuco, 2000.

\begin{tabular}{|c|c|c|c|}
\hline \multirow{2}{*}{ Faixa etária / Causas básica } & \multicolumn{2}{|c|}{ Mortalidade proporcional } & \multirow{2}{*}{$\begin{array}{c}\text { Coeficiente de Mortalidade Infantil Hopitalar* } \\
\% \text { \% }\end{array}$} \\
\hline & $\mathrm{n}$ & $\%$ & \\
\hline \multicolumn{4}{|l|}{ Neonatal (0 - 27 dias) } \\
\hline Reduzíveis & 198 & 75,3 & 54,2 \\
\hline Outra situação & 65 & 24,7 & 17,8 \\
\hline Sub-total & 263 & 100,0 & - \\
\hline \multicolumn{4}{|l|}{ Pós-neonatal (28 dias - 1 ano) } \\
\hline Reduzíveis & 181 & 60,1 & 49,5 \\
\hline Outra situação & 120 & 39,9 & 32,8 \\
\hline Sub-total & 301 & 100,0 & - \\
\hline \multicolumn{4}{|l|}{ Infantil (0 - 1 ano) } \\
\hline Reduzíveis & 379 & 77,4 & 103,7 \\
\hline Outra situação & 185 & 22,6 & 50,6 \\
\hline Total & 564 & 100,0 & - \\
\hline
\end{tabular}

* Coeficiente de mortalidade infantil hospitalar = razão entre o $n .^{\circ}$ de óbitos em menores de um ano e o total de internamentos (3.656) multiplicado por 1.000 


\section{Discussão}

As informações obtidas neste estudo são particularmente importantes. Apesar de serem provenientes de um único hospital, este é uma unidade de grande porte, cuja abrangência transcende o município onde está localizado, sendo de referência para o estado de Pernambuco.

Do total de mortes infantis registradas no HGP/IMIP em 2000, $33 \%$ foram de crianças procedentes da capital, $36 \%$ da região metropolitana, excetuando-se a capital, Recife, e $31 \%$ do interior do estado, segundo o relatório institucional do Núcleo de Epidemiologia. 25 No mesmo ano, Pernambuco registrou 4.862 óbitos em menores de um ano. 26 Nesse período a capital contava com uma população geral de 1.422.905 habitantes, 1525.428 nascidos vivos residentes 27 tendo sido registrados 509 óbitos nesse grupo etário, conforme o Plano Municipal de Saúde do Recife. 28

O HGP/IMIP apresentou um obituário de menores de um ano maior que o dos residentes no Recife no ano 2000, em decorrência do seu nível de complexidade, pelas características dos serviços oferecidos e da clientela assistida. Esse número de mortes hospitalares é ainda mais elevado, pois foram excluídos do estudo os óbitos neonatais ocorridos na maternidade da Instituição.

Tais dados revelam a magnitude do evento em menores de um ano no HGP, uma vez que o serviço respondeu por praticamente $12 \%$ dos óbitos estaduais nesse grupo etário.

Uma das vantagens da realização do estudo em uma instituição com as características desta, além das expostas acima, se deve à confiabilidade dos dados, visto que todas as Declarações de Óbitos passam pelo processo de revisão sistemática, tendo o prontuário médico "Padrão ouro". Desta forma, praticamente não existe DO com causa básica mal definida, casos reservados aos pacientes que chegam sem vida ao serviço, sem esclarecimento médico, ou aqueles provenientes do domicílio. Na literatura consultada há referência quanto à imprecisão da causa básica razão pela qual alguns autores 17,19,22 têm submetido as DO ao julgamento de experts visando melhorar a qualidade do "Atestado médico".

$\mathrm{Na}$ avaliação das mortes infantis hospitalares, o cerne da questão está na identificação das causas, cujos óbitos poderiam ser evitados por medidas de atenção à saúde. ${ }^{7,23}$ Deve-se ressaltar que os autores do presente trabalho não desconhecem os determinantes socioeconômicos da mortalidade infantil, mas se apóiam na perspectiva do que os serviços de saúde poderiam fazer para reduzi-la.
No entanto, algumas considerações metodológicas merecem ser feitas quanto ao critério de classificação adotado e quanto ao indicador selecionado. Vários estudos realizados em alguns estados do Brasil, São Paulo, Rio de Janeiro, Pernambuco e Maranhão, já validaram estes critérios, 3,12,17,19-22 que se baseiam nos avanços técnico-científicos, independentemente de estarem disponíveis para toda a população. Por essa razão tais critérios são criticados, uma vez que desconsideram o estágio de desenvolvimento dos municípios e a oferta dos serviços de saúde loco-regionais. No entanto, se contra-argumenta a importância da construção de "imagem objetivo", a ser vislumbrada a partir da viabilidade de um sistema nacional de saúde, com acesso e qualidade da atenção adequada. 3

Além de existirem poucos trabalhos classificando as mortes intra-hospitalares quanto à evitabilidade, há diferenças também nas características das unidades como: número de leitos; tipo de prestador público, filantrópico ou privado; perfil da população assistida, serviços oferecidos e gravidade dos casos; diferentes níveis de complexidade e tecnologias disponíveis. 23

Em que pese às dificuldades relatadas, os resultados revelaram informações interessantes que sugerem uma baixa resolutividade do sistema de saúde, uma vez que $75 \%$ das mortes neonatais e $60 \%$ das pósneonatais ocorreram por causas reduzíveis por medidas de atenção à saúde.

Em se tratando dos óbitos neonatais, o estudo realizado em 1994 por Coutinho 19 nas cinco maiores maternidades do Recife, em número de internamentos e em nível de complexidade, revelou que $85,1 \%$ dos óbitos poderiam ter sido evitados, uma vez que $57 \%$ aconteceram por causas reduzíveis e $28 \%$ por causas parcialmente reduzíveis, resultados esses próximos aos do presente estudo, seis anos depois (75\% por causas reduzíveis). Esses achados foram corroborados por Sarinho, 20 em 1995, ampliando o estudo para o universo dos óbitos da cidade do Recife, onde encontrou $78,8 \%$ das mortes neonatais precoces e $69,6 \%$ das tardias que poderiam ter sido reduzidas por adequado controle da gravidez, atenção apropriada ao parto, diagnóstico e tratamentos precoces, ou pelas parcialmente reduzíveis.

Percentuais elevados de mortes evitáveis também obteve Ribeiro e Silva, ${ }^{21}$ em São Luís, Maranhão, quando analisaram uma série histórica de óbitos neonatais (1979 a 1996) utilizando os mesmos critérios, encontrando mais da metade por causas reduzíveis. As informações obtidas em todos esses trabalhos sugerem a ocorrência de mudanças pouco significativas no sistema de saúde e no padrão de vida 
da população.

A diferença entre os estudos de Sarinho 20 e de Ribeiro e Silva ${ }^{21}$ e este se encontra na população analisada, pois naqueles os dados se referem ao município, enquanto no presente trabalho, são provenientes de um único hospital; porém os resultados são concordantes: a maioria dos óbitos neonatais do IMIP ocorreu por causas reduzíveis por diagnóstico e tratamento precoces ou pelas parcialmente reduzíveis. Dentre as primeiras estavam, principalmente, sepse, síndrome de aspiração de mecônio e pneumonias congênitas, enquanto para as parcialmente reduzíveis, o maior percentual foi devido à síndrome do desconforto respiratório.

Segundo alguns autores, $3,13,29$ procedimentos de baixo custo e alta efetividade da rotina dos serviços poderiam ser implementados sem, necessariamente, maiores investimentos em tecnologias complexas, e assim, muitos óbitos evitados.

A baixa proporção de óbitos reduzíveis por adequado controle da gravidez e atenção ao parto deve-se talvez a procedência da amostra, pois as crianças vieram de outras maternidades ou de seus domicílios, fato que pode ser considerado como um viés de seleção. Associado a isso houve dificuldade na identificação das causas básicas maternas, por não constarem do prontuário médico. Isso se explica pela ausência de encaminhamentos esclarecedores e/ou da genitora acompanhando as transferências, conforme observado em um estudo sobre condições de transporte dos recém-nascidos admitidos na instituição. 30

Quanto à mortalidade proporcional pós-neonatal por causas reduzíveis identificou-se um percentual menor que o encontrado para a cidade do Recife, em 1995 e para Brejo da Madre de Deus e Bom Conselho (interior do estado de Pernambuco), em 1999.17,22 Enquanto nesses municípios variou de $71,4 \%$ a $92,2 \%, 17,22$ o IMIP apresentou $60 \%$. Mesmo considerando as diferentes dimensões populacionais (hospital/município), já referidas anteriormente, esse resultado provavelmente está associado às características do hospital que é referência para as malformações congênitas, classificadas como "Não evitáveis" as quais totalizaram $24 \%$ dos casos.

A maior contribuição dentre as evitáveis verificou-se no grupo de "Outras reduzíveis" (44,2\%), que tem, entre outras doenças constantes desse grupo, as infecciosas intestinais e as do aparelho respiratório. Essas doenças são passíveis de controle e tratamento no nível local e se diagnosticadas precocemente, poderiam ser evitados o agravamento, o internamento e o óbito.

Desde a década de 80, o Ministério da Saúde, reconhecendo a importância dessas causas para a mor- talidade infantil e a factibilidade de seus controles, implantou o Programa de Assistência Integral à Saúde da Criança (PAISC), composto de cinco ações básicas: crescimento e desenvolvimento, aleitamento materno e orientação para o desmame, controle e assistência das doenças diarréicas, controle e assistência das infecções respiratórias agudas e imunização. ${ }^{31}$

Atualmente esse programa está incorporado à estratégia da Atenção Integrada às Doenças Prevalentes da Infância (AIDPI), adotada pelo Ministério da Saúde nos meados dos anos 90, que sistematiza e aborda todas as ações básicas fornecendo atendimento intergral à criança menor de cinco anos. Direcionada aos profissionais que atuam nas unidades de atenção primária à saúde, a AIDPI orienta para o manejo dos casos, identificação dos sinais de gravidade e na educação às famílias para os cuidados em casa. 32

Pelo nível de complexidade do hospital aqui estudado seria pouco esperada a ocorrência de um número elevado de mortes pelas doenças mencionadas. Assim as altas taxas de mortalidade encontradas sugerem problemas de acesso ou de qualidade na assistência desde o nível primário de atenção. Também poderia supor-se que os programas de base comunitária, Saúde da Família (PSF) ou de Agentes Comunitários de Saúde (PACS), não apresentem a cobertura populacional esperada ou não estejam cumprindo com um de seus objetivos, que é a vigilância aos grupos de risco.

O coeficiente de mortalidade infantil hospitalar por causas reduzíveis por medidas de atenção à saúde foi bastante elevado, 103,7\%o internamentos. Essa taxa não pode ser comparada às dos estudos realizados no Recife17 (21,85\%o nascidos vivos, no ano de 1995), em Brejo da Madre de Deus e em Bom Conselho (39,8 e 63,7\%o nascidos vivos, em 1999), 22 visto que as populações utilizadas para o cálculo foram diferentes: no presente estudo considerou-se o número de internamentos no ano, enquanto nos demais o número de nascidos vivos no mesmo período.

Diante dos resultados apresentados, tendo como base os critérios de evitabilidade de 1991, esse continua sendo útil na identificação dos óbitos por causas evitáveis, em que pese a nova versão, revisada à luz dos novos conhecimentos técnicos-científicos. ${ }^{12}$ A nova classificação tem a desvantagem de ser mais rigorosa, visto que outras doenças, a exemplo de algumas malformações, foram incorporadas às causas reduzíveis, porém exigem tratamentos com tecnologias mais sofisticadas, não disponíveis para a grande maioria da população infantil, que persiste adoecendo e morrendo por diarréia e pneumonia, doenças típicas da pobreza. 


\section{Agradecimentos}

Ao Convênio Centro Colaborador IMIP e ao CENEPI (Centro Nacional de Epidemiologia) do Ministério da Saúde.

\section{Referências}

1. Rutstein DD, Berenberg W, Chalmers TC, Child CG, Fishman AP, Perrin EB. Measuring the quality of medical care: a clinical method. N Engl J Med 1976; 294: 582-8.

2. Hartz ZMA, Champagne F, Leal MC, Contandriopoulos AP. Mortalidade infantil "evitável" em duas cidades do Nordeste do Brasil: indicador da qualidade do sistema local de saúde. Rev Saúde Pública 1996; 30: 310-8.

3. Leal MC. Evolução da mortalidade infantil no estado do Rio de Janeiro na década de 80: o componente neonatal [tese doutorado]. Rio de Janeiro: Escola de Saúde Pública da Fundação Oswaldo Cruz; 1996.

4. Araújo BF, Bozzetti MC, Tanaka ACA. Mortalidade neonatal precoce no município de Caxias do Sul: um estudo de coorte. J Pediatr [Rio de Janeiro] 2000; 76: 200-6.

5. Monteiro CA. Contribuição para o estudo do significado da evolução do coeficiente da mortalidade infantil no $\mathrm{Mu}$ nicípio de São Paulo, SP (Brasil) nas três últimas décadas (1950-1979). Rev Saúde Pública 1982; 16: 7-18.

6. Barros FC. Infant mortality in Southern Brazil: a population based study of causes of death. Arch Dis Child 1987; 62: 487-90

7. Victora FC. Epidemiologia da desigualdade: um estudo longitudinal de 6000 crianças brasileiras. 2.ed. São Paulo: Hucitec; 1989.

8. Barros FC. The Pelotas birth cohort study, 1982-1987: strategies for following up 6,000 children in a developing country. Perinat Pediatr Epidemiol 1990: 4: 267-82.

9. UNICEF (Fundo das Nações Unidas para Infância). Tabelas estatísticas. In: UNICEF Situação mundial da infância: 1989. Brasília (DF) O Fundo; 1989. p. 90-3.

10. Monteiro CA, Zuñiga HPP. Uma nova hipótese para a ascensão da mortalidade infantil da cidade de São Paulo nos anos 60. In: Monteiro CA, organizador. Velhos e novos males da saúde no Brasil. São Paulo: Hucitec; 1995. p. 157-72.

11. UNICEF (Fundo das Nações Unidas para Infância). Tabelas estatísticas. In: UNICEF. Situação mundial da infância: 1998. Brasília (DF) O Fundo; 1998. p. 92-7.

12. Ortiz LP. Características da mortalidade neonatal no estado de São Paulo [tese doutorado]. São Paulo: Faculdade de Saúde Pública da Universidade de São Paulo; 1999.

13. Carvalho ML. Mortalidade neonatal e aspectos da qualidade da atenção à saúde na Região Metropolitana do Rio de Janeiro [dissertação mestrado]. Rio de Janeiro: Escola Nacional de Saúde Pública da Fundação Oswaldo Cruz; 1993.

14. RIPSA (Rede Interagencial de Informações para a Saúde).
Indicadores de dados básicos para a saúde: IDB 2001. Brasília (DF): Ministério da Saúde; 2001. Disponível em: www.datasus.gov.br [2002 ago 7].

15. FIBGE (Fundação Instituto Brasileiro de Geografia e Estatística). Censo 2000. Rio de Janeiro: A Fundação; 2000. Disponível em: www.ibge.gov.br/home/estatistica /populacao/censo [2002 out 28].

16. Molina CAG, López MVL. Mortalidad evitable. El caso de la Frontera Norte de México, 1980-1990. Cad Saúde Pública 1995; 11: 395-407.

17. Guimarães MJB. Mortalidade infantil e condições de vida: uma análise da desigualdade espacial no Recife [dissertação mestrado]. Recife: Instituto Materno Infantil de Pernambuco; 1998.

18. Leite AJM, Marcopito LF, Diniz RLP, Silva AVS, Souza LCB, Borges JC, Carmo e Sá, HL. Mortes perinatais no município de Fortaleza, Ceará: o quanto é possível evitar? J Pediatr [Rio de Janeiro] 1997; 73: 367-82.

19. Coutinho SB. Mortalidade neonatal em cinco maternidades da cidade do Recife, 1994: relatório final de pesquisa apresentado ao UNICEF. Recife: UNICEF; 1996.

20. Sarinho SW. Mortalidade neonatal na cidade do Recife: um estudo de caso-controle [tese doutorado]. Recife: Centro de Ciências da Saúde da Universidade Federal de Pernambuco; 1998.

21. Ribeiro VS, Silva AAM. Tendências da mortalidade neonatal em São Luís, Maranhão, Brasil, de 1979 a 1996. Cad Saúde Pública 2000; 16: 508-14.

22. Frias PG. Análise de implantação do projeto de redução da mortalidade infantil em dois municípios de Pernambuco com semelhantes condições de vida [dissertação mestrado]. Recife: Centro de Ciências da Saúde, Departamento Materno Infantil da Universidade Federal de Pernambuco; 2001.

23. Travassos C, Noronha JC, Martins M. Mortalidade hospitalar como indicador de qualidade: uma revisão. Ci Saúde Col 1999; 4: 367-81.

24. OMS (Organização Mundial da Saúde). Classificação internacional de doenças. 4. ed. 10. rev. São Paulo: Editora da Universidade de São Paulo; 1998.

25. IMIP (Instituto Materno Infantil de Pernambuco), Núcleo de Epidemiologia. Relatório. Bol IMIP 2001.

26. Ministério da Saúde. FUNASA (Fundação Nacional de Saúde). Banco de Dados dos Sistemas de Informação sobre Mortalidade (SIM) e Nascidos Vivos (SINASC): 1996 a 2000.[CD-ROM]. Brasília (DF): O Ministério; 2000.

27. Recife. Secretaria de Saúde. Crianças do Recife: perfil de 
nascimentos, 1995-2000. Cad Ciclo Vida [Série Criança] 2002; (1).

28. Recife. Secretaria de Saúde. Plano municipal de saúde: 2001. Recife: A Secretaria; 2001

29. Lansky S, França E, Leal MC. Mortes perinatais em Belo Horizonte, Minas Gerais, 1999. Cad Saúde Pública 2002; 18: $1389-400$.

30. Silveira SMM. Hipotermia na admissão: fator de risco para mortalidade em recém-nascidos transferidos para o Instituto Materno Infantil de Pernambuco [dissertação mestrado]. Recife: Instituto Materno Infantil de Pernambuco; 2001.

31. Vidal SA, Frias PG, Marques NM. Avaliação normativa das ações do Programa de Atenção Integrada à Saúde da Criança (PAISC). Rev Bras Saúde Matern Infant 2001; 1: 129-35.

32. Benguigui Y. Integrated management of childhood illness (IMCI): an innovative vision for child health care. Rev Bras Saúde Matern Infant 2001; 1: 223-36.

Recebido em 9 de abril de 2003

Versão final reapresentada em 30 de junho de 2003

Aprovado em 4 de agosto de 2003 